\title{
"Recent Advances in the Manipulation of Circularly Polarised Light with Cellulose Nanocrystal Films."
}

Authors: S.N. Fernandesa, ${ }^{*}$ L.F. Lopesb, M.H. Godinhoa,*

ai3N/CENIMAT, Department of Materials Science, Faculty of Science and Technology, Universidade NOVA de Lisboa, Campus de Caparica, 2829-516, Caparica, Portugal.

b Museu Nacional de História Natural e da Ciência \& Centre for Ecology, Evolution and Environmental Changes (cE3c), University of Lisbon, Rua da Escola Politécnica, 58, 1250102 Lisboa, Portugal

Corresponding authors: M.H. Godinho (mhg@fct.unl.pt) and S.N. Fernandes (sm.fernandes@fct.unl.pt)

\begin{abstract}
Significant advances have been made to control the iridescence and the selective reflection of left circularly polarised (LCP) light, and transmission of right circularly polarised (RCP) light of solid films prepared from cellulose nanocrystals (CNCs). However the manipulation of the photonic properties of the CNCs films, which reflect both RCP and LCP light is less investigated. Solid films prepare from natural sources as CNCs have advantageous characteristics that are absent in other synthetic structures, such as wide availability and renewability. Here we review and compare recent research activity involving the production and characterization of photonic band gap structures resulting from an anisotropic layer inserted between two cholesteric layers with different helical pitches but the same handedness. We make connections between systems existing in Nature and synthetic ones with the hope of advancing in the production and manipulation of CNCs-based photonic structures.
\end{abstract}




\section{Keywords:}

Cellulose nanocrystals

Iridescence

Circularly Polarised Light

Liquid Crystals

Bio-inspired materials

Photonics

\section{Introduction}

Anisotropic structures, mainly shaped from cellulose micro/nano rods, are often found outside the cells of plants. At the genesis of these solid structures can be a liquid crystalline phase that subsequently solidifies due to chemical reactions of crosslinking and mineralization [1]. The main liquid crystalline organization of cellulose-based structures, also existing in plant tissues, corresponds to different arrangements of a cholesteric liquid crystalline phase. In this sense the first part of the work will be dedicated to a brief introduction to liquid crystals, namely to cholesteric and nematic structures [2].

A common characteristic of liquid crystals (LCs) is that they are formed by anisometric entities with one or two characteristic dimensions much larger than the third. These objects, with synthetic or natural origin, can be molecules, nano- and microparticles, which are organized and exhibit the optical anisotropy of crystals and the mechanical properties (fluidity) of liquids.

In order to describe the cholesteric arrangements found for cellulose nanocrystals (CNCs) rods it is useful to briefly address the main characteristics of the nematic LC structure. The nematic phase, of a material formed by rods of nanoparticles, presents the centres of gravity 
of the nanorods randomly distributed, with no positional order, such as in an isotropic liquid, but with orientational order for long distances compared to the dimensions of the nanorods.

In order to specify the orientation of the nanorods at a given point of the nematic phase a unit vector, $\mathbf{n}$, named director $[3,4]$ is used. As the orientation of the anisometric particles is not perfect, due to fluctuations, a degree of order, $\langle S\rangle$, is defined by

$$
<S>=\frac{1}{2}<3 \cos ^{2} \theta-1>
$$

where $\theta$ specifies the angle that the major axis of a generic rod makes with the director and $<$ $\cdots>$, takes into account the average value of the expression in parenthesis for a large number of rods. The value of $S$ varies between 0 and 1 in the nematic phase [3-5], being zero in the isotropic fluid and equal to one in the crystalline phase.

A cholesteric phase can be described as a nematic with a uniform natural twist around a normal axis to the director as schematically shown in Fig. 1, with the pitch, $\mathrm{P}$, of the structure greater than the length of the rods [6]. The planes usually used in literature to represent the nematic chiral structure [7] only help to better visualize the helical structure of the medium but do not correspond to any discontinuity in the arrangement of the rods.

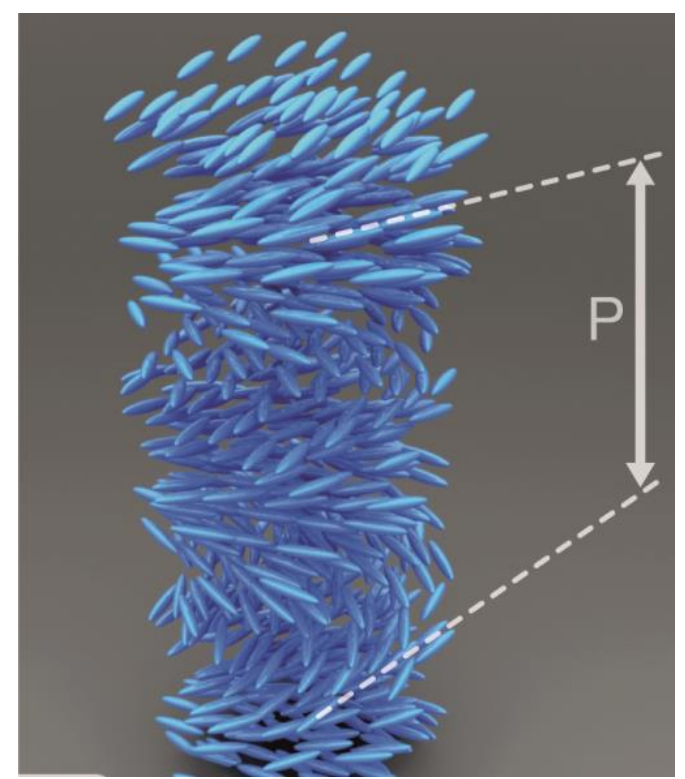


Fig. 1. Scheme of the cholesteric arrangement of the nano rods, in blue, (the helical structure of the medium has no discontinuity). In a distance $\mathrm{P}$ (measured along the optical axis) the main axis of the nano rods, in the pseudo-nematic layers, rotate by an angle of $2 \pi$. From [6] Reprinted by permission of Taylor \& Francis Ltd.

The natural twisting of the cholesteric phase is usually attributed to the chirality of the constituent particles [1, 8], which modifies the intermolecular forces responsible for the orientational order and produces the helical distortion. The origin of the formation of a helical arrangement for $\mathrm{CNCs}$ is still under debate. However, the helical cellulose-based configuration results in remarkable optical and mechanical properties, which gives the material multiple possibilities of practical applications.

For example, in plants the helicoidal cellulose structures promote the mechanical resistance of the walls of the cells allowing long cells to be flexible but resistant to bending forces [1]. In addition, the cholesteric arrangements in the solid state are responsible for the brilliant colours observed, for example, in the fruit of Pollia genus plants [9]. The wavelength reflected by the structure, $\lambda_{o}$, its colouration, varies upon the value of the pitch, the view angle and the average refraction index of the material, $\bar{n}=\frac{n_{e}+n_{o}}{2}\left(n_{e}\right.$, extraordinary and $n_{o}$, ordinary refraction indexes of the medium), according to the expression,

$\lambda_{o}=\bar{n} P \sin \theta$

where $\theta$ is the angle between the incident light and the cholesteric layers [2,7].

The rotatory power of the cholesteric structure changes with the wavelength of the incident light. Let us consider the value of $\theta$ equal to $\frac{\pi}{2}$, which means that the wavelength is reflected by the structure along its helical axis. The incident circularly polarised (CP) light, with the same handedness as the cholesteric structure, behaves similar to a periodic media. When the values of the wavelengths are in the width of the reflection band according to $\Delta \lambda=\Delta \mathrm{nP}$ around $\lambda_{o}$, the cholesteric structure reflects the circular component of the polarised light of the same handedness of the helical structure and transmits the other component. This 
reflection is similar to the Bragg reflection observed for the X-rays, in a periodic structure with a distance equal to $\mathrm{P} / 2$. If $\lambda_{o}$ is in the visible region of the spectrum and the cholesteric is illuminated with white light, $50 \%$ of the light intensity in the $\Delta \lambda$ band will be reflected, whereas outside this band gap the light will be transmitted. As the band is relatively narrow the cholesteric will then present a bright coloration. Higher-order reflections appear and the reflection band shifts when the values of $\theta$ deviate from $\frac{\pi}{2}$.

This brief review is mainly dedicated to summarise the work involved with the optical properties of CNCs-based solid iridescent films with selective reflection and transmission of circularly polarised light. Special emphasis will be given to bio inspired materials with photonic properties that are manipulated by external fields, which add additional features to the systems found in animals and plants.

\section{Circularly polarised light}

In nature, most of the cholesteric structural colours have its origin in left-handed helical cholesteric arrangements [1, 10-14]. Beautiful examples are the exocuticles of some beetles, as in Cetonia aurata (Linnaeus, 1758) (Fig. 2 a-c). Chitin nanorods that can be seen in the scanning electron microscopy (SEM) photo, Fig. 2 d, are the structural units of the cholesteric arrangement. In addition to the brilliant colours that the cholesteric structure can offer to the materials the right- or left- handed helical arrangement translates to different responses of the material when observed with CP filters. The left-handed helical structure present in C. aurata beetle is responsible for the extinction of colour when observed with a right circularly polariser (RCP) as shown in Fig. 2 c. 

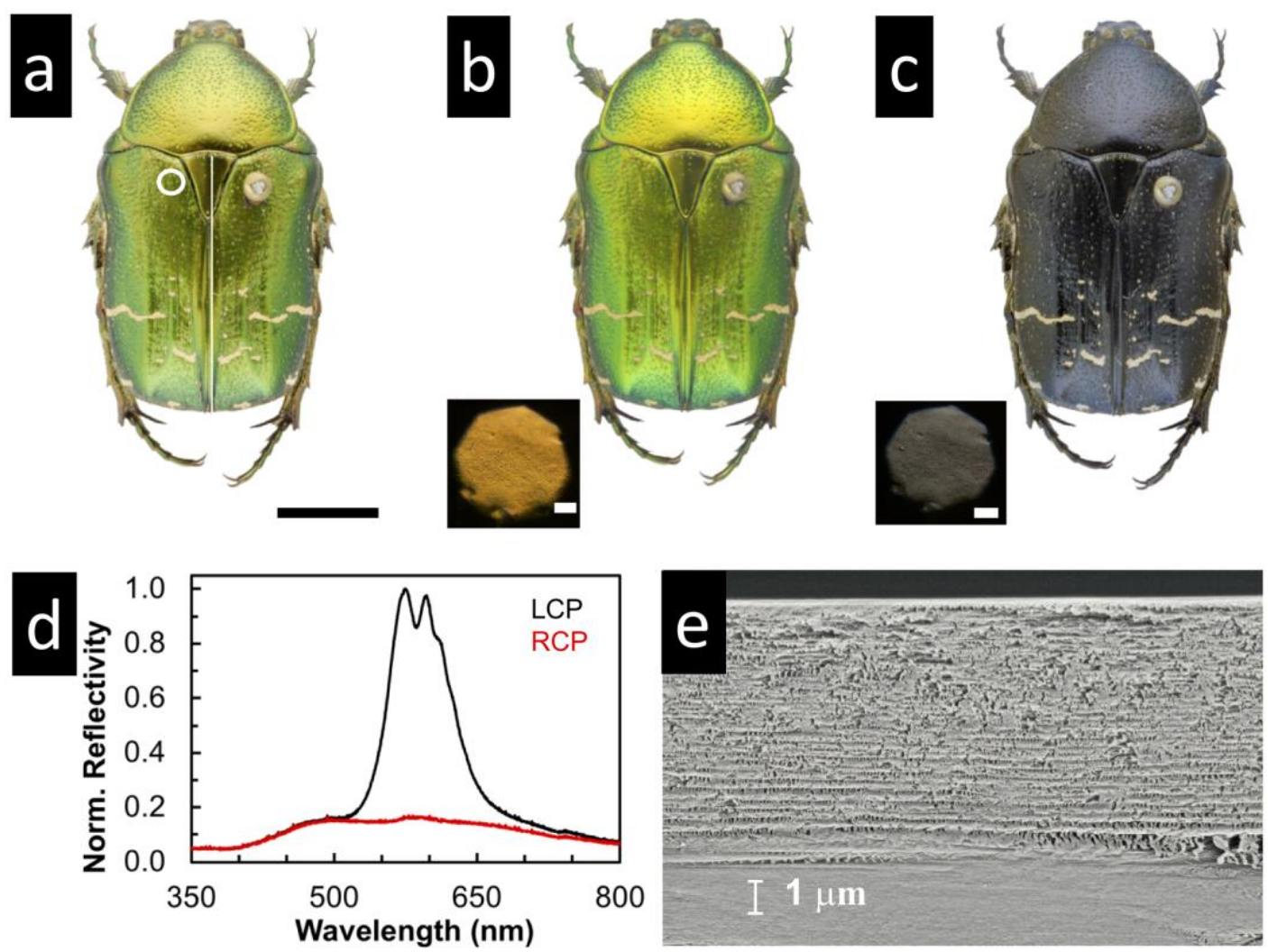

Fig. 2. Photographs of the beetle $C$. aurata a) and b) bright yellow and green colours as seen in unpolarised light or with a left circular polariser, respectively. c) Extinction of colours when observed with a right circular polariser. d) Reflectance spectra of the exocuticle of the C. aurata beetle for both LCP and RCP light channels (a-d: unpublished work). e) Scanning electron microscopy (SEM) micrograph of a section cut vertically through the cuticle of the beetle showing the lamellar organization in the exocuticle responsible for its bright coloration [15]. (C) 2013 Optical Society of America. Scale bars represent a) $0.5 \mathrm{~cm}$, inset in b, c) $100 \mu \mathrm{m}$.

However, reflections on left circularly polariser (LCP) and RCP channels were also observed in natural systems, such as in the exocuticle of the species Chrysina resplendens (Boucard, 1875) (Fig. 3 a -c) and in the outer cellulosic layers of the fruit of Pollia condensata C.B. Clarke [9]. The similar photonic response of the cholesteric structures of Pollia and $C$. resplendens is associated to the presence of a helical arrangement of nanorods either from cellulose or chitin respectively, but the response in the RCP channel has different origins. While $P$. condensata. produces cellulose-based left- and right-handed helicoidal arrangements that coexist in neighbouring cells, the exocuticle of $C$. resplendens presents only left-handed helical structures. 
The optical reflection characteristics of the $C$. resplendens exocuticle are due to a sandwich structure in which an anisotropic layer is confined between two left solid cholesteric arrangements, as show in Fig. 3 (a-d). The anisotropic layer plays the role of a half-wave phase retarder. While the colour reflected by the exocuticle depends on the pitch of the helicoidal structures, the average refraction of the medium and view angle, the anisotropic layer plays a crucial role to the RCP light channel response [2].

The scheme shown in Fig. 3 d) illustrates the effect of the $\lambda / 2$ retardation plate between two different left cholesteric structures. Circular polarisers were used to observe the sample with incident light perpendicular to the film cholesteric layers (Fig. 3 b-c). The light with lefthanded circular polarisation is reflected by the cholesteric structure at the top of the film (Fig. $3 \mathrm{~b}$ ), while the right-handed circular polarisation is transmitted (Fig. 3 c). The right-handed circular polarisation changes into left-handed due to the anisotropic layer that acts as a $\lambda / 2$ retardation plate promoting a phase shift:

$$
\Delta \phi=2 \pi \frac{\Delta n h}{\lambda}
$$

where $\mathrm{h}$ is the anisotropic layer thickness and $\Delta n=n e-n o$ its birefringence. Then, the converted left-handed CP light travels along the anisotropic layer until reaching the surface of the second cholesteric left-handed structure that reflects left circular polarised light, which is again converted by the retardation plate into right-handed polarised light and transmitted by the cholesteric existing at the top surface of the film. Therefore, both LCP and RCP light are reflected by the material $[2,7,10,16]$. The reflected wavelengths on LCP and RCP light channels depend on the values of the pitches of the two cholesteric arrangements. 


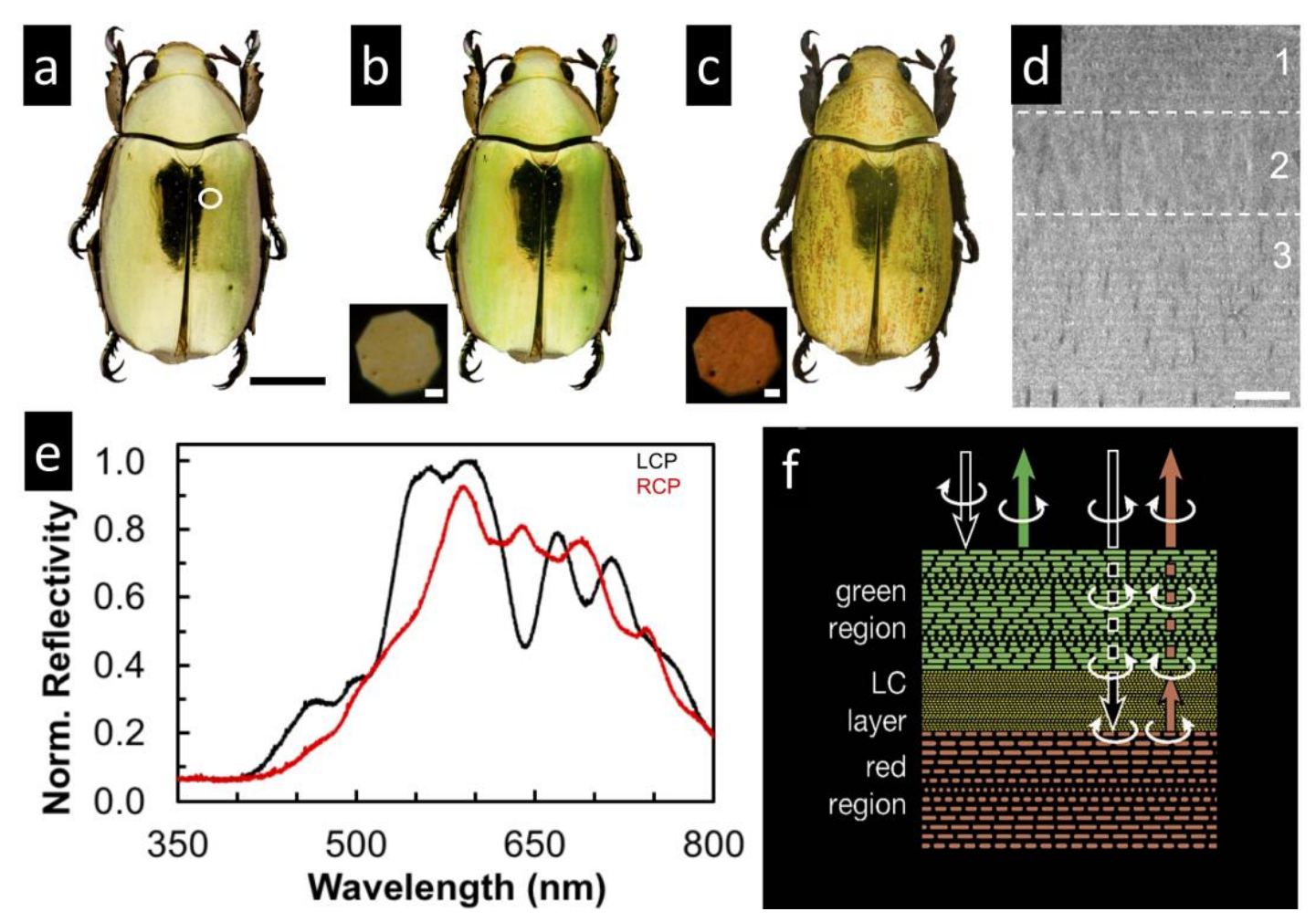

Fig. 3. Photographs of the C. resplendens beetle a) Bright yellow and greenish colours as seen in unpolarised light. b) Yellow and a more evident green colours seen with a left circular polariser. c) Yellow and orange colours observed with a right circular polariser. d) Transmission electron microscopy (TEM) micrograph of a section cut vertically through the cuticle of the beetle showing two lamellar organizations (1 and 3) separated by an anisotropic layer (2). e) Reflectance spectra of the exocuticle of the C. resplendens exocuticle for both LCP and RCP light channels (a-d: unpublished work). f) Scheme of the sandwiched structure (an anisotropic layer (yellow) between two cholesteric structures with the same handedness but different values of the pitch (in green and red)), reflectance and transmittance optical characteristics of a film with the anisotropic layer acting as a $\lambda / 2$ plate. Panel f adapted with permission [7]. Copyright 201, Wiley-VCH. Scale bars represent: a) $0.5 \mathrm{~cm}$, inset in a,b) $50 \mu \mathrm{m}$, d) $1 \mu \mathrm{m}$.

Structural colours in insects have many perceived functions, such as crypsis, aposematism and mate signalling [17-21]. The function of circular polarised (CP) light reflected by insects is not yet understood. Some authors published interesting but hypothetical work. It was proposed that selective circular polarised light reflection can be a way to enhance the contrast between objects and their background [22], providing a secret communication channel, for example, to conspecific sexual signalling, that could not be perceived by their vertebrate predators $[23,24]$. 
This idea was reinforced by the observation [25] that stomatopod crustaceans are able to detect and discriminate the handedness of CP light. Furthermore these authors have been able to associate a behavioural response with this capacity. They hypothesise this to be a sexual signalling feature, since these species reflect $\mathrm{CP}$ light in the visible spectrum region usually used for sexual display and are present only in males.

In insects, several specimens of scarab beetles (Coleoptera, Scarabaeidae) are well known to reflect LCP light, while LCP and RCP light were found to be reflected in the case of $C$. resplendens [26]. Whether or not these insects are able to discriminate CP light is still to be proved.

Recently, behavioural response to $\mathrm{CP}$ light has been detected in Chrysina gloriosa (LeConte, 1854) suggesting that they are capable of distinguishing CP light [23]. However, another behavioural study [27], was unable to detect a response to CP light stimuli in 4 species of scarab beetles (Anomala dubia (Scopoli, 1763), Anomala vitis Fabricius, 1775, Cetonia aurata (Linnaeus, 1758), Protaetia cuprea (Fabricius, 1775)). Together with the fact that CP light reflection is similar in both male and female, led the authors to hypothesize that CP light reflection is just a by-product of the cholesteric arrangement in the beetle cuticle and that its main function is more probably a mechanical or chemical one, conferring the cuticle resistance against mechanical or chemical stress.

These two studies were done with insect species with different distribution (North America and Europe, respectively) and distinct habitats. C. resplendens inhabits a further distinct habitat, living in tropical areas in Central America. It is possible that according with habitat and lifestyle CP light reflection may have different effects in intraspecific communication. Even if the main sexual attractant in beetles are pheromones, being able to discriminate $\mathrm{CP}$ light signals may further help to contrast their mates from the circularly unpolarised foliage environment. 


\section{Mimicking the optical response of natural structures}

\subsection{Left-circularly polarised light response}

Circularly polarised filters are used to detect the handedness of the cholesterics and it was found that iridescent cellulosic films, prepared from CNCs, reflect LCP light and transmit RCP light when illuminated with white light (Fig. 4 a and b). SEM pictures revealed a periodic structure along the films' cross section (Fig. 4) indicating the presence of a cholesteric structure. The distance between two successive layers corresponds to P/2. This value can be compared with the maximum wavelength reflected by the material with incident light perpendicular to the cholesteric layers (spectra given in Fig. 4).

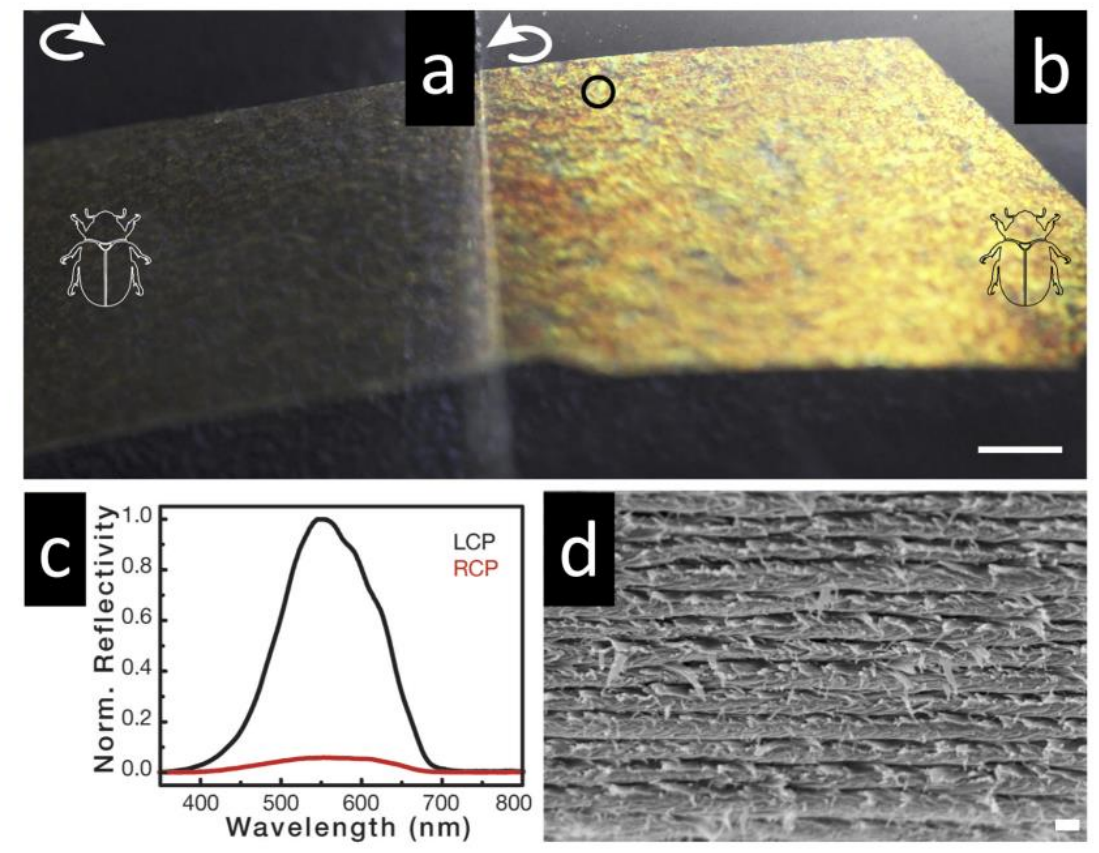

Fig. 4. Photographs of a CNC film. a) Extinction of colours when observed with a right circular polariser. b) Bright colours as seen with a left circular polariser. c) Reflectance spectra of the film for both LCP and RCP light channels. d) Scanning electron microscopy (SEM) micrograph of the cross section of the film showing the cholesteric organization responsible for its bright coloration when illuminated by white light and seen in the 
LCP channel. Scale bars represent: a) and d) 200nm. Panel c adapted with permission [7]. Copyright 2016, Wiley-VCH.

The study of the remarkable optical properties, as the iridescent colours, observed in Fig. 4, which arises from the $\mathrm{CNC}$ hierarchically self-assembled, when the solvent of the cholesteric liquid crystalline CNCs suspension is slowly evaporated, has been widely explored in the past decade by the scientific community. Nonetheless, the full control of this LC phase is far from being totally established. The interesting results obtained so far confirmed that the cholesteric structure retained in the solid state is influenced by a myriad experimental conditions. Among these are the source of cellulose, its type and concentration of acid, time and temperature of the hydrolysis process, which translate in the ionic strength and size of the resultant CNCs. The concentration of CNCs in the suspension, as well as the energy given to the system by the ultrasonic treatment and the solvent evaporation rate are other factors that one has to have in mind when producing such cholesteric structures from $\mathrm{CNCs} /$ water suspension. Several works have been published where these issues are addressed $[1,6,28$ 36]. The control of the optical properties and mainly the maximum wavelength reflection by adjusting the cholesteric pitch value has been widely investigated and several approaches have been tested. For instance, it is possible to induce changes in the pitch values by adding electrolytes (such as sodium chloride), glucose, compatible polymers, or by applying electric or magnetic fields to the CNC suspension [37-42]. Whilst the CNCs properties have been substantially investigated, their use in the preparation of well-aligned cholesteric structures and their selective reflection to CP light are still far from being fully explored.

In the past six years, $\mathrm{CNCs}$ liquid crystalline phase has been used as cholesteric templating of inorganic compounds, such as silica, and/or metallic nanoparticles, through a process named by the authors as evaporation induced self-assembly (EISA), followed by thermal removal of the CNC [43-48]. With this technique, new applications for the mesoporous cholesteric 
materials with strong structural coloration started to be surveyed. For instance, the same methodology allowed Shopsowithz et al. [49] to produce a refractive index sensor, by exploiting the loss of iridescence verified on porous cholesteric silica film while it was submersed in water. The use of inorganic materials as part of the matrix of the film could even give rise to interesting chiral nematic mesoporous cellulose films if instead of the CNC the silica is removed, thus enabling the researchers to use them in stabilizing gold nanoparticles suspensions [50].

Composite systems where the authors combine polymers or surfactants with cholesteric CNC have been the subject of some recent works. In these, the chiral optical properties of the CNC films are maintained while coupled with the properties of such organic compounds, conferring the final structure with enhanced functionalities, such as thermo-responsiveness, flexibility, or conductivity [42, 51-53]. For instance, Espinha et al. were able to produce a shape memory photonic reflector while coupling the $\mathrm{CNC}$ with an elastomer, the polydiociltrate [51]. The photonic reflectors produced from the infiltration of iridescent CNC films with the elastomer presented a shift of the reflected wavelength to red or infrared regions of the electromagnetic spectrum. After curing the material, the authors could observe that the CNC film was totally encased by elastomer layers. Despite this fact the composite system maintained the circular polarisation properties by reflecting LCP light and transmitting RCP light. The presence of the elastomer allows for the production of a shape memory system by programming its shape above the melting transition of the polymer $\left(\sim 30^{\circ} \mathrm{C}\right)$.

Another interesting type of transparent depolarising optical filter was also produced from CNCs solid films. It was shown that iridescent CNCs films, with response in the left channel, can not only depolarise incident light but also the optical response cab be manipulated rendering them transparent when illuminated with white light [54]. A scheme illustrating the 
mechanism behind this observation is given in Fig. 5. While a cholesteric iridescent film, prepared from CNCs (CF1), reflects the LCP light and transmits the RCP light (Fig. 5 a) a completely different response is obtained when transparent films with no cholesteric arrangements (quasinematic) (CF5 and CF6) are placed in the pathway of the light, between the observer and the sample CF1. The CF5 and CF6 films convert RCP and LCP light into unpolarised light acting as depolarising filters (Fig. 5).
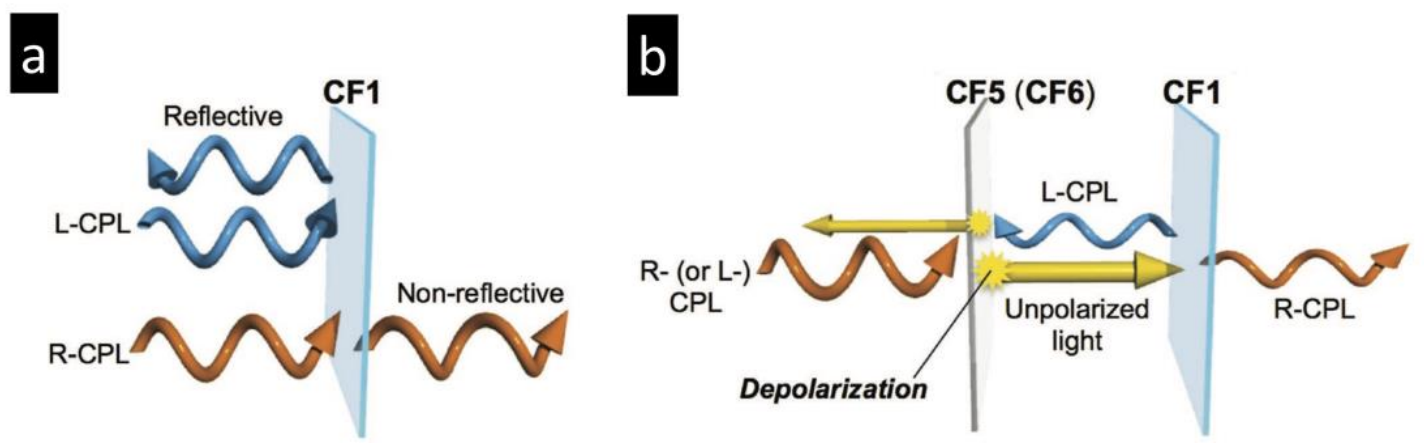

Fig. 5. a) Schematic representation of RCP and LCP light reflection and transmission, a) through an iridescent CNC film (CF1) and in b) for a layered sample consisting of a film with a quasinematic organization (CF5 and CF6) and CF1. Adapted with permission [54]. Copyright 2017, Wiley-VCH.

The previous examples do not take into account the existence of a polydomain cholesteric structure, translated into a width of the reflection band of $\sim 100 \mathrm{~nm}$ (Fig. $4 \mathrm{c}$ ), far from the expected width observed for an ideal well organized helically modulated structure. However, the width of the reflection band of these films produce until is not large enough for them to be considered as broadband reflectors.

Cao et al. took advantage of these features and cleverly engineered a system were by adding a specific amount of sub-micrometric micellar surfactant, into CNCs cholesteric suspensions (Fig. 6 a), it was possible to produce a solid structure with broadband circular polarisation [55]. By SEM observations, the authors demonstrated the existence of the aforementioned characteristic CNCs left-handed cholesteric arrangement characteristic of the $\mathrm{CNC}$, but with a 
highly dispersed distance between layers (from 120nm to $400 \mathrm{~nm}$ ) along the cross-section of the films. The white scattering film produced (Fig. 6 b) enables the reflection of LCP light (Fig. 6 c) and the transmission of RCP light (Fig. 6 d). These results were also confirmed by the transmission circular dichroism (CD) and UV-Vis spectroscopy, where the positive signal (indicative of the left-handedness helicity) and a wide range of wavelength reflection confirms the production of a broadband left circular polarising film (Fig. 6 e). This work opened the opportunity for using this type of cholesteric arrangements to produce optical filters, polarisers and coatings.[55]
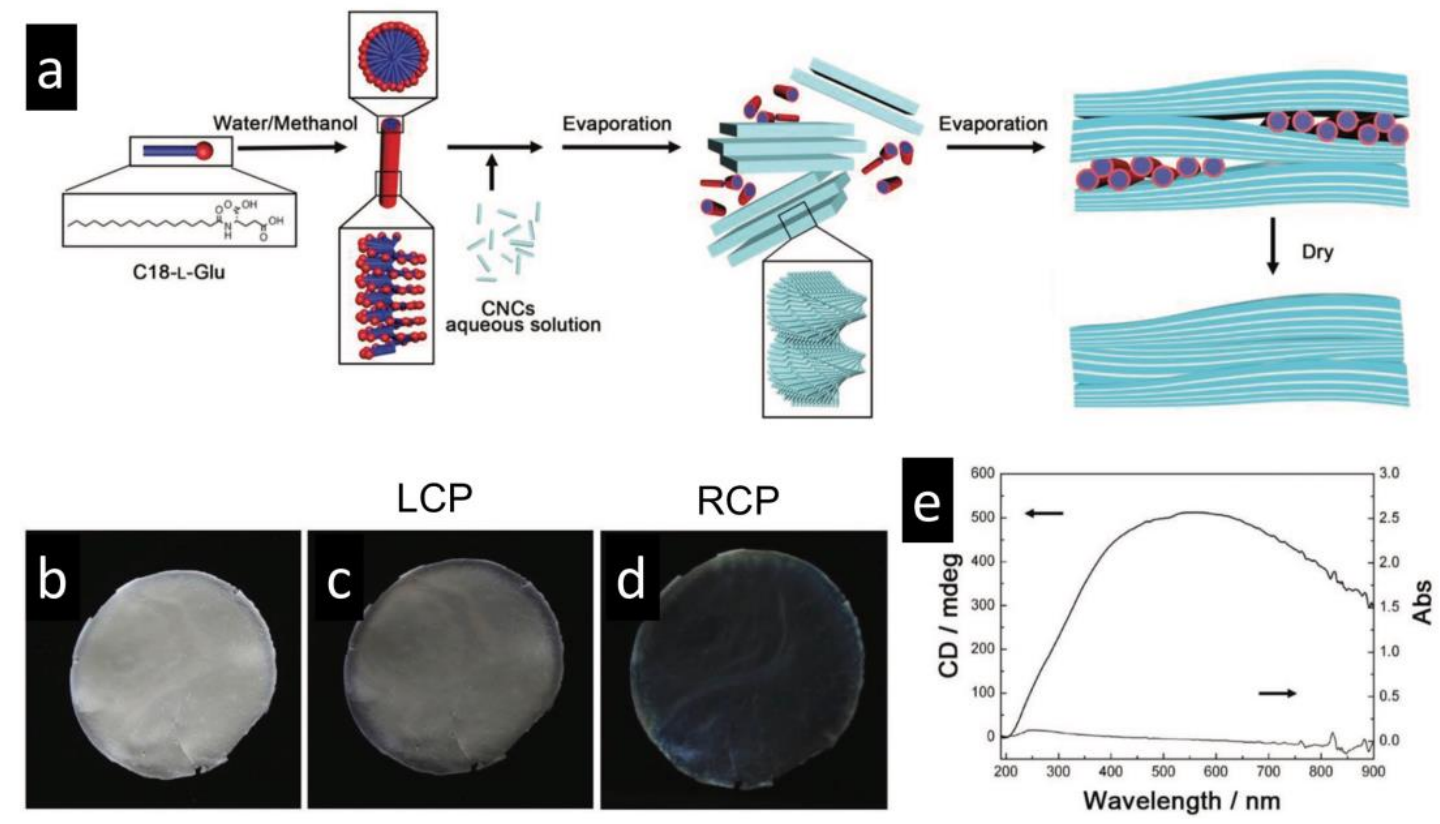

Fig. 6. a) Schematic process of the distortion of the cholesteric arrangement of the CNC aqueous suspension as the solvent evaporates due to the presence of micelles of $\mathrm{C}_{18}-\mathrm{L}-\mathrm{Glu}$. b-d) Photographs of the produced solid CNC film observed b) with natural light, where the strong white structural colouration can be seen, the same reflection of light is seen c) with a LCP polariser, however when the film is viewed with a RCP filter d) a dark appearance is observed due to the transmission of light. e) CD and UV-Vis spectra for the CNC film confirming the production of a broadband LCP film. Adapted with permission [55]. Copyright 2018, Wiley-VCH.

\subsection{Right and Left circularly polarised light response}

The cholesteric solid films produced from $\mathrm{CNCs}$ described until now present a $\mathrm{CP}$ optical 
response mechanism similar to the one observed for the cuticle of the $C$. aurata beetle. Exploring this material to produce new photonic structures that mimic the diverse CP optical response of $C$. resplendens is still challenging, however some promising results have been obtained.

In fact, photonic structures, which simultaneously reflect LCP and RCP light have been produced from synthetic materials, and more recently from cellulose-based materials. To be able to produce materials with such optical characteristics one can develop a system composed of an anisotropic layer, which acts as a half wave retardation plate, sandwiched between two self-assembled cholesteric liquid crystalline layers with the same handedness, similar to the exocuticle of $C$. resplendens, or prepare the photonic material from cholesteric films where both helicity senses coexist, as observed in the case of the $P$. condensate fruit. An interesting example of a synthetic system can be found in the work of Matranga et al. [56]. The authors were able to mimic the golden structural colouration of the $C$. resplendes with LCP and RCP light reflection by combining different self-organised and self-aligned synthetic liquid crystal polymers, Fig. 7. In a skilful way, they were able to produce a freestanding system that consists of layers of UV photo-crosslinked reactive mesogens (RM), which present LC phases, coated on the top of each other. Each layer promotes the appropriate alignment for the following layer confirmed by the transmittance spectra presented in Fig. 7 a. The several spectra depict the evolution of the transmittance along the producing process, consisting of a sequence of depositions on a rubbed polyimide layer starting with a cured nematic layer, which follows the alignment of the rubbing. Followed by a single cholesteric $(\mathrm{Ch})$ layer that aligns wells leading to a good reflector (Fig. 7 a, Ch spectrum). Then a deposition of an untwisted nematic layer that acts as a half-wave retarder (Fig. 7 a, $\mathrm{Ch}+\mathrm{HW}$ spectrum), and after that a second cholesteric layer (Fig. 7 a, $\mathrm{Ch}+\mathrm{HW}$ spectrum). The transmittance of this self-aligned system is comparable to the one obtained 
when two separate Ch layers are joined together (Fig. 7 a, $\mathrm{Ch}+\mathrm{HW}+\mathrm{Ch}$ separate spectrum), proving the principle of self-organisation raised by the alignment of the underneath layer.

The colouration observed from the cuticle of the $C$. resplendens was remarkable reproduced using this process, as can be seen in the photograph presented in Fig. 7 b. However, instead of just one cholesteric layer they align two, with different pitch values (of $639 \mathrm{~nm}$ and $562 \mathrm{~nm}$ for the first and second layer, respectively). The retardation plate, subsequently deposited, was designed to work at a wavelength of $595 \mathrm{~nm}$, and was followed by another two cholesteric layers (at $562 \mathrm{~nm}$ and $639 \mathrm{~nm}$ ). In a cross-section SEM image of this structure one can observe this multilayer system (Fig. 7 c) [56]. The deposition of cholesteric layers with different pitches was the authors' solution to increase the width of the selective reflection band of these layers, and increase the similarity with the beetle reflections. However this also leads to an increase of the complexity of the multilayered system. Other systems derived from synthetic organic mesogens can be found in the literature where both left-handed and right-handed cholesteric arrangements coexist [8, 57]. Nevertheless, these systems are greatly dependent on synthetic costly materials. The preparation of these types of photonic structures based on natural materials, and specifically on the self-assembly of CNCs onto an aqueous liquid crystalline phase, is very attractive due to the affordable sample cost and myriad potential applications, such as in anti-counterfeit security systems and optical sensors. 

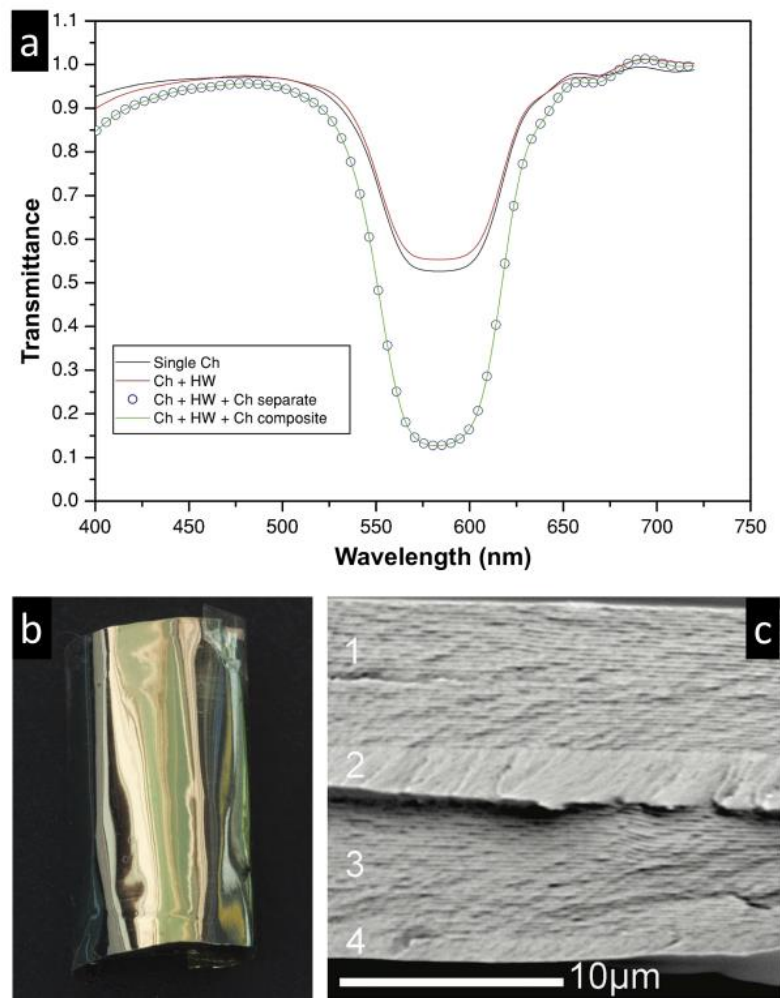

Fig. 7. Transmittance spectra evolution of the multilayer system of self-organising and self-aligned liquid crystal polymers consisting of a sequence of depositions: cured nematic layer onto polyimide rubbed layer, followed by a single cholesteric (Ch). Second deposition of a nematic layer that acts as a half-wave retarder (HW), and after second cholesteric layer ( $\mathrm{Ch}+\mathrm{Hw}+\mathrm{Ch}$ composite), with transmittance comparable to that of two separate Ch layers plus a HW layer stacked together $(\mathrm{Ch}+\mathrm{Hw}+\mathrm{Ch}$ separate). b) Reflector obtained from the RM polymeric liquid crystals that mimics the $C$. resplendens structural coloration. c) SEM cross-section image of the film shown in $\mathrm{b}$ where it is possible to see the two Ch regions ( 1 and 3 , composed by two different cholesteric sub-layers of 562 and $639 \mathrm{~nm}$ ), the half wave retardation layer (2) and the nematic alignment layer (4). Adapted with permission [56]. Copyright 2012, Wiley-VCH.

Recently, $\mathrm{Wu}$ et al. were able to mimic the optical $\mathrm{CP}$ reflection that arises from the exocuticle of the $C$. resplendens beetle with a system based on a composite material consisting of two CNCs cholesteric films and a polyethylene glycol diacrylate (PEGDA) film sandwiching a uniaxial oriented $25 \mu$ m polyamide-nylon 6 (PA-6) layer [16]. In this system, the authors deposit a layer of CNCs/PEGDA composite suspension on one side of the PA-6 film allowing the cellulosic composite to self-assemble into a cholesteric structure while the solvent slowly evaporates in a control environment. After complete solvent evaporation, the 
process was repeated on the other side of the PA- 6 layer. The obtained cholesteric layers were then photo-crosslinked. The resultant flexible structure presents a green metallic coloration that could be seen with both left-handed and right-handed circular polarisers, analogous to what is described by Kinoshita et al. [2]. The authors succeeded in tuning the maximum wavelength reflection of the cholesteric structures from the blue to the red region of the visible electromagnetic spectrum, by increasing the quantity of the PEGDA in the composite system, from 30 to $60 \%$ (wt./wt.). The same effect was achieved later by Zao et al. [42] with the composite system CNCs/polyethylene glycol (PEG). The appearance of the material is attributed to the increase of the pitch value of the cholesteric arrangement due to the presence of the polymer. Wu et al. were also able to demonstrate that the presence of this $\lambda / 2$ retarder in this type of device more than doubled the intensity of the reflected light, when compared with a bilayer cholesteric film system.

On another work an iridescent cholesteric CNCs film was impregnated with a nematic liquid crystal at room temperature giving rise to a composite material with LCP and RCP light reflections (Fig. 8 a,b) [7], being the nematic liquid crystal used the 4-cyano-4'pentylbiphenyl (5CB). To obtain such a composite system, the authors took advantage of a micro-gap existing between two top cholesteric layers along the cross-section of the CNC film, and filled it with a nematic liquid crystal (Fig. 8 c). 

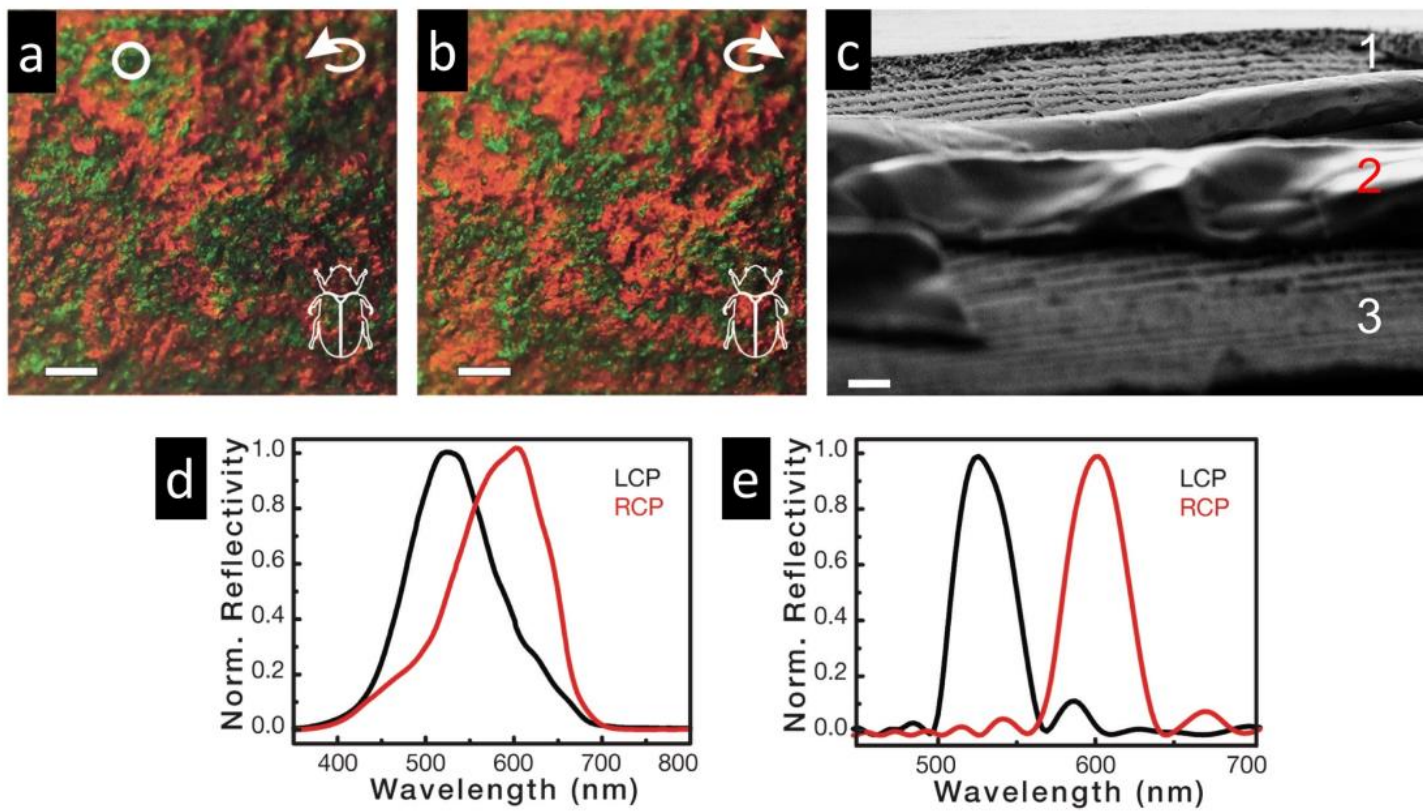

Fig. 8. Photographs of the $\mathrm{CNC} / 5 \mathrm{CB}$ composite system taken under $\mathrm{CP}$ light showing reflection in both a) LCP and b) RCP light channels, mimicking the C. Resplendens optical response (silhouette). SEM image of a crosssection of a CNC film filled with an anisotropic layer of 4CB (2) between two cholesteric layers (1 and 2) with different pitch values. Reflection spectra from LCP and RCP channels d) obtained experimentally that are in good agreement to what was e) simulated with a 4x4 Berreman's Matrix for this composite system. The scale bars in a-d represent $20 \mu \mathrm{m}$. Adapted with permission [7]. Copyright 2016, Wiley-VCH

A possible explanation for the observed result is the following: the cellulose matrix induces planar alignment on the nematic liquid crystal and this anisotropic layer acts as a $\lambda / 2$ wave retardation plate. The phase shift due to the nematic LC material was found responsible for the RCP reflection optical response. The optical characteristics of the CNC film, namely the values of the pitches along the cross section, were tuned in order to fulfil the requirements of equation (3). Fig. 8 d,e) shows the reflection spectra obtained with LCP and RCP polarisers, with the maximum reflection centred at $518 \mathrm{~nm} 610 \mathrm{~nm}$, respectively, and with a spectral width higher than $140 \mathrm{~nm}$.

\section{Manipulation of LCP and RCP light response of CNCs-based photonic films}

In the last composite system referred to above, made from iridescent $\mathrm{CNC}$ film with the $5 \mathrm{CB}$, and taking into account that the half-wave retarder is a liquid crystal, the authors were able to 
tune the reflected LCP and RCP light optical response by changing the orientation and the birefringence of the nematic LC phase, which were achieved by varying the temperature of the system above the nematic to isotropic (N-I) transition temperature, or by applying an electric field [7]. The use of temperature was previously employed by Manning et al. in an iridescent cholesteric glass, templated from the EISA liquid crystalline CNC suspension, and impregnated with the nematic liquid crystal 4'-n-octyl-4-cyanobiphenyl (8CB). In this work, the authors were able to control, in an on/off manner, the iridescence of the glass when the temperature of the composite was above the LC N-I transition temperature [58]. This result is due to the change in the index refraction of the LC phase in the composite system, equation 2 , giving rise to an increase in the cholesteric pitch value, and in this example, resulting in a loss of the macroscopically observed structural colouration. In the $\mathrm{CNC} / 5 \mathrm{CB}$ composite the use of temperature generates a decrease of the order parameter, $\leq \mathrm{S}>$, and a shift to higher pitch values of the reflected wavelength when observed with LCP channel above the N-I transition (Fig. 9 a to b) shown in the reflection spectra depicted in Fig. 9 g). Above the 5CB N-I transition temperature the composite system transmits light when observed with the RCP polariser (Fig. $9 \mathrm{c}$ to d) and a loss of the iridescence is observed, confirmed by the low reflectivity value obtained (Fig. 9 h). The schemes presented in Fig. 9 e,f) gives the reader a better understanding of the effect of the increase in temperature of the retardation layer, and the authors attributed this behaviour to the 5CB planar anchoring on the cellulose matrix [7]. 

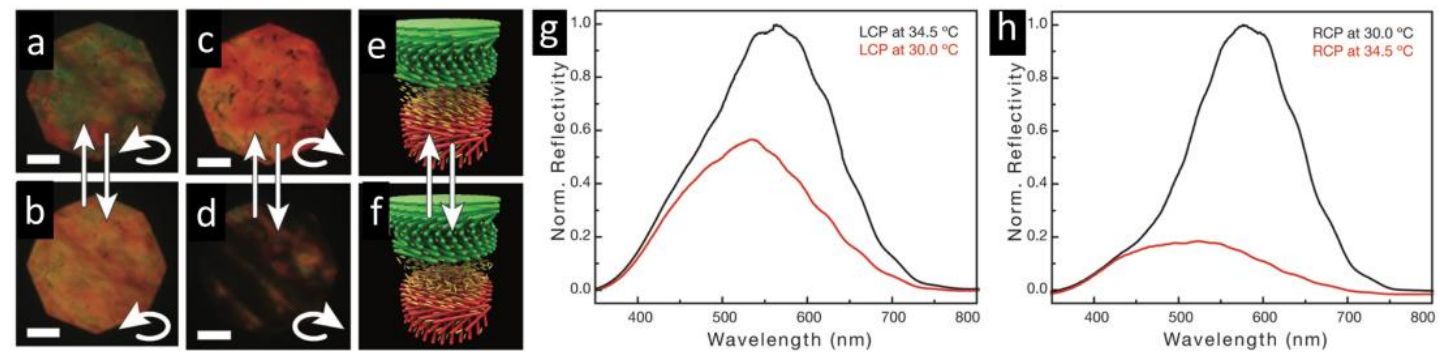

Fig. 9. a-b) LCP and c-d) RCP light reflection microphotographs of CNC/5CB composite system when submitted to a temperature rise above the 5CB N-I transition (b,d; T=34.5 $5^{\circ}$ ). Schemes showing the crosssection of the system with the half wave retardation layer below e) and above $f$ ) the N-I temperature transition. g) LCP and h) RCP light spectra obtained at the two temperatures. The spectra confirming that that above the NI temperature transition of the 5CB the composite system does not reflect the light in the RCP light channel. The scale bars in a-d represent $100 \mu \mathrm{m}$. Adapted with permission [7]. Copyright 2016, Wiley-VCH.

When mounted between two conductive indium tin oxide (ITO) coated glasses and supplemented with the proper electric contacts, the authors were able to apply an electric field and change the birefringence of 5CB. When this stimulus was applied the optical response of the second cholesteric layer (below the $\lambda / 2$ retarder) was seen, by observation of the colour variation from green to red in the LCP (Fig. 10 a- b) channel. If the device is observed with the RCP channel while the stimulus is applied the light transmission was achieved (Fig. $10 \mathrm{c}-\mathrm{d}$ ). The optical response of the device is reversible and the change in the birefringence of the $\lambda / 2$ layer occurs in just a few milliseconds. The cross-section schemes of the device in OFF and ON states (Fig. 10 e and f, respectively) presented by the authors, suggested that the $5 \mathrm{CB}$ will align in the direction of the electric field due to its positive dielectric anisotropy. The numerical simulation, by means of a 4x4 Berreman's Matrix, of the reflection spectra when changing the $5 \mathrm{CB}$ birefringence while the electric field is applied (Fig. $10 \mathrm{~g}$ ) is in concordance with the experimental results obtained (Fig. $10 \mathrm{~h}$ ) above a threshold value of $0.6 \mathrm{~V} \mu \mathrm{m}-1$ [7]. This work demonstrated that using this system, besides 
being able to tune the $\mathrm{CP}$ optical response, one can also adjust the reflectivity by applying an electric field.
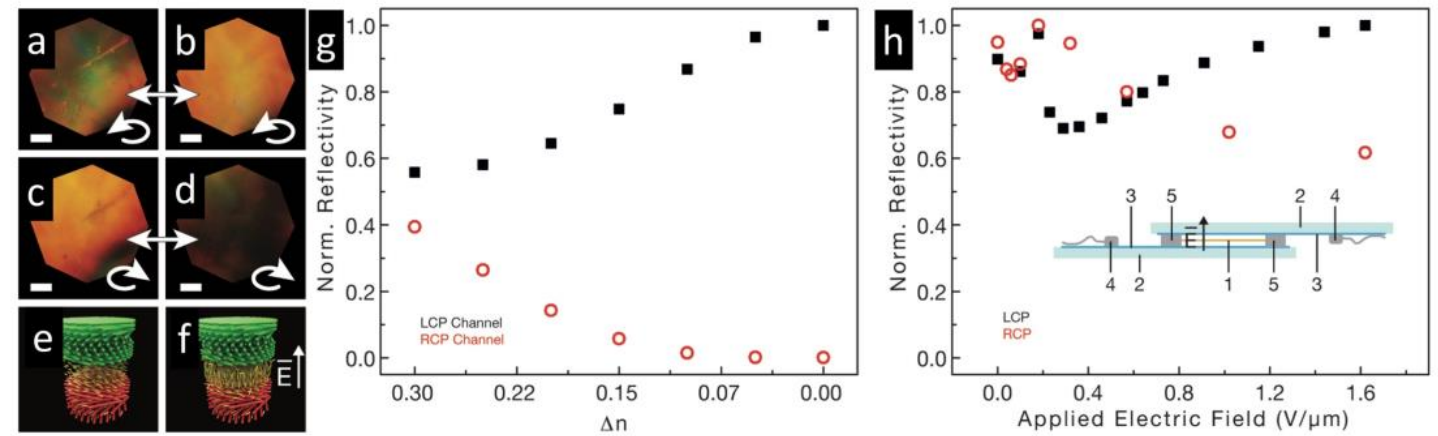

Fig. 10. a-b) LCP and c-d) RCP light reflection microphotographs of CNC/5CB composite when submitted to an electric field (b,d; $E=1.5 \mathrm{~V} \mu \mathrm{m}-1)$ perpendicular to the $5 \mathrm{CB}$ positive dielectric layer. Schemes showing the anisotropic layer $(\lambda / 2)$ e) without and $\mathrm{f}$ ) with the application of the electric field. Variation of the reflectivity of $\mathrm{CNC} / 5 \mathrm{CB}$ composite $\mathrm{g}$ ) in function of the $5 \mathrm{CB}$ birefringence simulated by $4 \mathrm{x} 4$ Berreman's Matrix $\mathrm{h}$ ) in function of the applied electric field obtained experimentally for both LCP and RCP light channels. The inset in $\mathrm{h}$ depicts the electro-optical cell (1- composite system; 2-glass substrate; 3- ITO and alignment layer; 4-electric contacts; 5-spacers. The scale bars in a-d represent $20 \mu \mathrm{m}$. Adapted with permission [7]. Copyright 2016, Wiley-VCH.

Having in mind the water-responsive behaviour of the cellulose nanocrystals-based systems previous observed by the MacLachlan group [59-61], Wu et al. studied the impact of relative humidity on the optical properties of the sandwiched structure of CNC/PEGDA/PA-6 mention above [16] (schematic diagram of production shown in Fig. 11 a). A remarkable bending deformation away from the direction of the applied external stimulus ( $80 \%$ relative humidity at $25^{\circ} \mathrm{C}$ ) was noted (Fig. $11 \mathrm{~b}$, initial and intermediate state), which was fully restored when the stimulus is removed. Accompanying this asymmetrical expansion, justified by the presence of the hydrophobic PA-6 layer between the two cholesteric layers, a change in the reflected structural colouration from green to red was observed (Fig. $11 \mathrm{~b}$, intermediate state). Taking advantage of this hydrophobic film between the cholesteric layers the authors were able to obtain a system with different colouration on each side, as long as the stimulus 
was applied or even the same colouration on both sides of the structure (if the stimulus is applied on both sides) (Fig. $11 \mathrm{~b}$, final state). This sequence of humidity application was followed by spectroscopy, and from the experimental reflection spectra (Fig. $11 \mathrm{c}$ ) it is possible to infer that the swelled states do not present RCP light reflection.

a
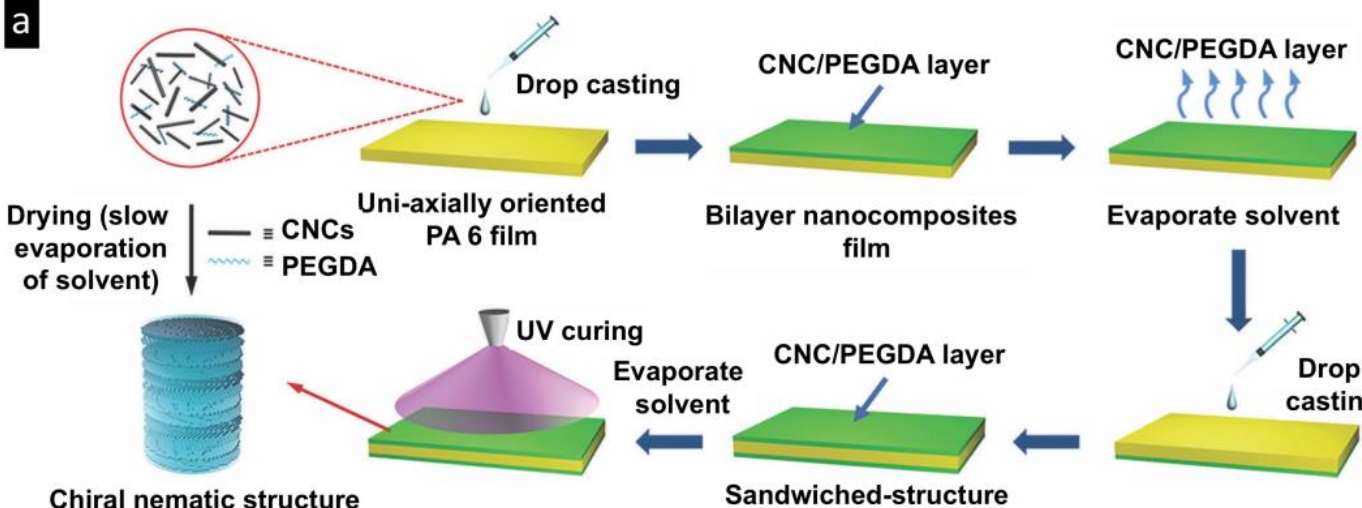

Bilayer nanocomposites

Evaporate solvent film

Evaporate solvent

CNC/PEGDA layer solvent

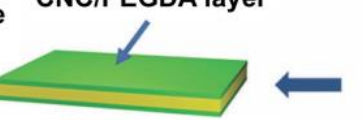

Sandwiched-structure nanocomposites films
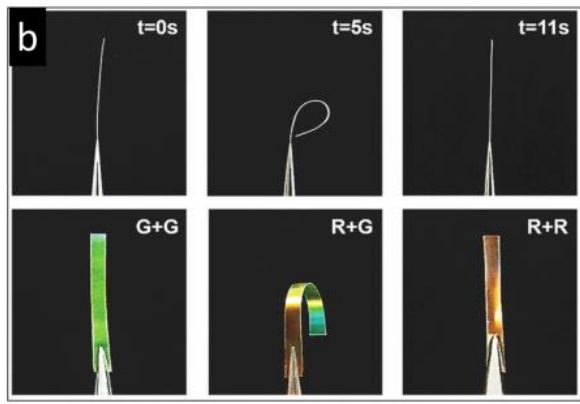

Initial State Intermediate State

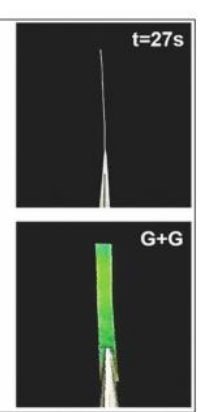

Initial State

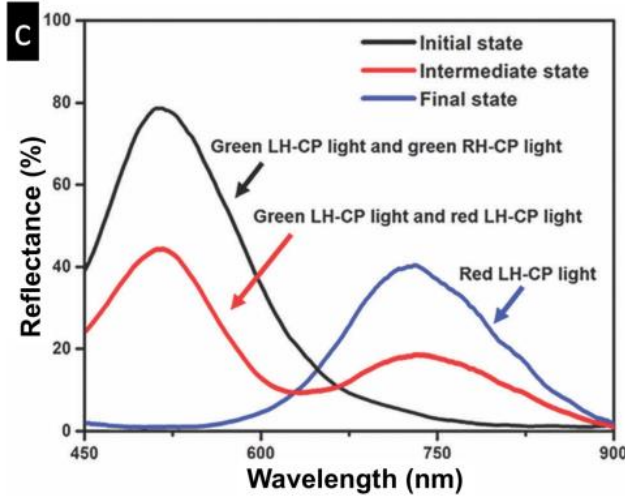

Fig. 11. Representation of the multi-step process to produce the hyper-reflective structure of two cholesteric CNC/PEGDA layers separated by a uniaxially oriented PA-6 film. b) Water-vapour induced bending deformation (upper photographs) and colour change (bottom row) of the system: initial state no stimulus; intermediate state with application of humidity on one side of the device; final state with both sides exposed to humidity. When the external stimulus is removed the system unbends and the colour and shape change to their initial state. c) Reflectance spectra of the sequence depicted in b) where it is noticeable that when the stimulus is applied the device does not reflect light on the RCP channels. Reproduced from [16] with permission from the Royal Society of Chemistry, Copyright 2016.

According to the author this is due to the fact that the PA-6 layer only acts as a half-wave retardation plate at the green region of the spectrum. Although not referred to in the article, 
one can notice that the application of humidity as an external stimulus led to a dynamic control of the CP reflection response of this system, which goes from presenting both LCP and RCP light reflection without the stimulus, to just LCP when the stimulus is applied. This system is very promising as a moisture photonic sensor.

\section{Conclusions}

In recent years a growing interest among the scientific community has been observed on the use of cellulose nanocrystals based materials, derived from its self-assembly into cholesteric liquid crystalline phases that can be frozen into iridescent solid structures. The optical characteristics of the films mimicked several structures found in Nature, namely those in some exocuticles of the beetles. A multitude of methods to control and optimize the photonic behaviour of the solid film have been reported. Special attention has been given to their feature of selective reflection, reflecting LCP light and transmitting RCP light. Some authors were even capable of producing broadband reflectors from CNCs films by inducing disorder in the cholesteric arrangements (quasinematic structures). However, all of these structures are only capable of reflecting LCP light, since the helical sense is ruled by the cholesteric liquid crystalline phase derived from the CNCs self-assembly. Nonetheless recently, interest in the production of CNC systems/devices that are able to reflect both LCP and RCP light channels has been emerging, mainly those mimicking the optical response observed for the bright $C$. resplendens beetle. Also of significance are the studies done so far showing that optical response of the iridescent CNCs films, produced by combination of polymer networks and LCs, can be controlled by application of an external stimuli.

These bioinspired materials, with additional functionalities, open the possibility of creating devices with tailor-made optical properties. The manipulation of the circularly polarised light optical response enables their use in a broader range of precise optical applications. 


\section{Acknowledgements}

This work is funded by FEDER funds through the COMPETE 2020 Program, National Funds through FCT - Portuguese Foundation for Science and Technology and POR Lisboa2020, under the projects with references POCI- 01-0145-FEDER-007688 (Reference UID/CTM/50025), UID/BIA/00329/2013， PTDC/CTM-BIO/6178/2014， M-ERANET2/0007/2016 (CellColor) and PTDC/CTM-REF/30529/2017. S.N. Fernandes and LF. Lopes acknowledge the Minister of Science, Technology and Higher Education for National Funds, European Social Funds and FCT for fellowships number SFRH/BDP/78430/2011 and SFRH/BPD/84478/2012, respectively. This work was supported by the Histology and Comparative Pathology laboratory [Electron Microscopy service] of the Instituto de Medicina Molecular João Lobo Antunes, specifically Andreia Pinto for her kind support. We would like to thank Carlos Silva for providing the $C$. chrysina specimen. The $C$. aurata specimen observed was provided by the Insect Collection of the National Museum of Natural History and Science, University of Lisbon.

\section{References:}

[1] M. Mitov, Cholesteric liquid crystals in living matter, Soft Matter 13(23) (2017) 4176-4209.

[2] S. Kinoshita, S. Yoshioka, J. Miyazaki, Physics of structural colors, Rep. Prog. Phys. 71(7) (2008) 076401.

[3] P.G. Gennes, J. Prost, Liquid Crystals: Main Types and Properties, The Physics of Liquid Crystals, Oxford University Press, Oxford, U.K., 1993, pp. 1-40. 
[4] P.G. Gennes, J. Prost, Cholesterics, The Physics of Liquid Crystals, Oxford University Press, Oxford, U.K., 1993, pp. 263-336.

[5] Y. Li, E. Prince, S. Cho, A. Salari, Y. Mosaddeghian Golestani, O.D. Lavrentovich, E. Kumacheva, Periodic assembly of nanoparticle arrays in disclinations of cholesteric liquid crystals, Proc. Natl. Acad. Sci. U.S.A. 114(9) (2017) 2137.

[6] J.P. Canejo, N. Monge, C. Echeverria, S.N. Fernandes, M.H. Godinho, Cellulosic liquid crystals for films and fibers, Liquid Crystals Reviews 5(2) (2017) 86-110.

[7] S.N. Fernandes, P.L. Almeida, N. Monge, L.E. Aguirre, D. Reis, C.L.P. de Oliveira, A.M.F. Neto, P. Pieranski, M.H. Godinho, Mind the Microgap in Iridescent Cellulose Nanocrystal Films, Adv. Mater. 29(2) (2017) 1603560.

[8] M. Mitov, Cholesteric liquid crystals with a broad light reflection band, Adv. Mater. 24(47) (2012) 6260-76.

[9] S. Vignolini, P.J. Rudall, A.V. Rowland, A. Reed, E. Moyroud, R.B. Faden, J.J. Baumberg, B.J. Glover, U. Steiner, Pointillist structural color in Pollia fruit, Proc. Natl. Acad. Sci. U. S. A. 109(39) (2012) 15712-5.

[10] V. Sharma, M. Crne, J.O. Park, M. Srinivasarao, Structural Origin of Circularly Polarized Iridescence in Jeweled Beetles, Science 325(5939) (2009) 449-451.

[11] V. Sharma, M. Crne, J.O. Park, M. Srinivasarao, Bouligand Structures Underlie Circularly Polarized Iridescence of Scarab Beetles: A Closer View, Mater. Today 1 (2014) 161-171.

[12] S. Vignolini, T. Gregory, M. Kolle, A. Lethbridge, E. Moyroud, U. Steiner, B.J. Glover, P. Vukusic, P.J. Rudall, Structural colour from helicoidal cell-wall architecture in fruits of Margaritaria nobilis, J. R. Soc. Interface 13(124) (2016).

[13] S. Vignolini, E. Moyroud, B.J. Glover, U. Steiner, Analysing photonic structures in plants, J. R. Soc. Interface 10(87) (2013) 20130394. 
[14] B.D. Wilts, H.M. Whitney, B.J. Glover, U. Steiner, S. Vignolini, Natural Helicoidal Structures: Morphology, Self-assembly and Optical Properties, Mater. Today 1 (2014) 177-185.

[15] H. Arwin, T. Berlind, B. Johs, K. Järrendahl, Cuticle structure of the scarab beetle Cetonia aurata analyzed by regression analysis of Mueller-matrix ellipsometric data, Opt. Express 21(19) (2013) 22645-22656.

[16] T. Wu, J. Li, J. Li, S. Ye, J. Wei, J. Guo, A bio-inspired cellulose nanocrystal-based nanocomposite photonic film with hyper-reflection and humidity-responsive actuator properties, J. Mater. Chem. C 4(41) (2016) 9687-9696.

[17] D.J. Kemp, M.E. Herberstein, G.F. Grether, Unraveling the true complexity of costly color signaling, Behav. Ecol. 23(2) (2012) 233-236.

[18] A.R. Parker, The diversity and implications of animal structural colours, J. Exp. Biol. 201(16) (1998) 2343-2347.

[19] K.L. Prudic, A.K. Skemp, D.R. Papaj, Aposematic coloration, luminance contrast, and the benefits of conspicuousness, Behav. Ecol. 18(1) (2007) 41-46.

[20] A. Sweeney, C. Jiggins, S. Johnsen, Polarized light as a butterfly mating signal, Nature 423 (2003) 31.

[21] B.D. Wilts, K. Michielsen, J. Kuipers, H. De Raedt, D.G. Stavenga, Brilliant camouflage: photonic crystals in the diamond weevil, Entimus imperialis, Proc. R. Soc. Lond. B. Biol. Sci. 279(1738) (2012) 2524-2530.

[22] T.W. Cronin, N. Shashar, R.L. Caldwell, J. Marshall, A.G. Cheroske, T.-H. Chiou, Polarization Vision and Its Role in Biological Signaling, Integr. Comp. Biol. 43(4) (2003) 549-558.

[23] P. Brady, M. Cummings, Differential Response to Circularly Polarized Light by the Jewel Scarab Beetle Chrysina gloriosa, Am. Nat. 175(5) (2010) 614-620. 
[24] E.J. Warrant, Polarisation Vision: Beetles See Circularly Polarised Light, Curr. Biol. 20(14) (2010) R610-R612.

[25] T.-H. Chiou, S. Kleinlogel, T. Cronin, R. Caldwell, B. Loeffler, A. Siddiqi, A. Goldizen, J. Marshall, Circular Polarization Vision in a Stomatopod Crustacean, Curr. Biol. 18(6) (2008) 429-434.

[26] E.D. Finlayson, L.T. McDonald, P. Vukusic, Optically ambidextrous circularly polarized reflection from the chiral cuticle of the scarab beetle Chrysina resplendens, J. R. Soc. Interface 14(131) (2017).

[27] M. Blahó, Á. Egri, R. Hegedüs, J. Jósvai, M. Tóth, K. Kertész, L.P. Biró, G. Kriska, G. Horváth, No evidence for behavioral responses to circularly polarized light in four scarab beetle species with circularly polarizing exocuticle, Physiol. Behav. 105(4) (2012) 1067-1075.

[28] A.P.C. Almeida, J.P. Canejo, S.N. Fernandes, C. Echeverria, P.L. Almeida, M.H. Godinho, Cellulose-Based Biomimetics and Their Applications, Adv. Mater. 30(19) (2018) 1703655

[29] D. Klemm, F. Kramer, S. Moritz, T. Lindstrom, M. Ankerfors, D. Gray, A. Dorris, Nanocelluloses: a new family of nature-based materials, Angew. Chem. Int. Ed. Engl. 50(24) (2011) 5438-66.

[30] J.P.F. Lagerwall, C. Schütz, M. Salajkova, J. Noh, J. Hyun Park, G. Scalia, L. Bergström, Cellulose nanocrystal-based materials: from liquid crystal self-assembly and glass formation to multifunctional thin films, NPG Asia Materials 6(1) (2014) e80.

[31] R.M. Parker, G. Guidetti, C.A. Williams, T. Zhao, A. Narkevicius, S. Vignolini, B. FrkaPetesic, The Self-Assembly of Cellulose Nanocrystals: Hierarchical Design of Visual Appearance, Adv. Mater. 30(19) (2018) 1704477. 
[32] C. Honorato-Rios, A. Kuhnhold, J.R. Bruckner, R. Dannert, T. Schilling, J.P.F. Lagerwall, Equilibrium Liquid Crystal Phase Diagrams and Detection of Kinetic Arrest in Cellulose Nanocrystal Suspensions, Front. Mater. 3 (2016).

[33] M. Ličen, B. Majaron, J. Noh, C. Schütz, L. Bergström, J. Lagerwall, I. DrevenšekOlenik, Correlation between structural properties and iridescent colors of cellulose nanocrystalline films, Cellulose 23(6) (2016) 3601-3609.

[34] J.H. Park, J. Noh, C. Schütz, G. Salazar-Alvarez, G. Scalia, L. Bergström, J.P.F. Lagerwall, Macroscopic Control of Helix Orientation in Films Dried from Cholesteric Liquid-Crystalline Cellulose Nanocrystal Suspensions, ChemPhysChem 15(7) (2014) $1477-1484$

[35] P.X. Wang, W.Y. Hamad, M.J. MacLachlan, Structure and transformation of tactoids in cellulose nanocrystal suspensions, Nat. Commun. 7 (2016) 11515.

[36] A.G. Dumanli, H.M. van der Kooij, G. Kamita, E. Reisner, J.J. Baumberg, U. Steiner, S. Vignolini, Digital Color in Cellulose Nanocrystal Films, ACS Appl. Mater. Interfaces 6(15) (2014) 12302-12306.

[37] S. Beck, J. Bouchard, R. Berry, Controlling the Reflection Wavelength of Iridescent Solid Films of Nanocrystalline Cellulose, Biomacromolecules 12(1) (2011) 167-172.

[38] B. Frka-Petesic, G. Guidetti, G. Kamita, S. Vignolini, Controlling the Photonic Properties of Cholesteric Cellulose Nanocrystal Films with Magnets, Adv. Mater. 29(32) (2017) 1701469.

[39] B. Frka-Petesic, H. Radavidson, B. Jean, L. Heux, Dynamically Controlled Iridescence of Cholesteric Cellulose Nanocrystal Suspensions Using Electric Fields, Adv. Mater. $29(11)(2017) 1606208$.

[40] D. Gray, Recent Advances in Chiral Nematic Structure and Iridescent Color of Cellulose Nanocrystal Films, Nanomaterials 6(11) (2016) 213. 
[41] X. Mu, D.G. Gray, Droplets of cellulose nanocrystal suspensions on drying give iridescent 3-D “coffee-stain” rings, Cellulose 22(2) (2015) 1103-1107.

[42] K. Yao, Q. Meng, V. Bulone, Q. Zhou, Flexible and Responsive Chiral Nematic Cellulose Nanocrystal/Poly(ethylene glycol) Composite Films with Uniform and Tunable Structural Color, Adv. Mater. 28(29) (2017) 1701323.

[43] G.R. Meseck, A.S. Terpstra, M.J. MacLachlan, Liquid crystal templating of nanomaterials with nature's toolbox, Curr. Opin. Colloid Interface Sci. 29 (2017) 9-20.

[44] K.E. Shopsowitz, H. Qi, W.Y. Hamad, M.J. Maclachlan, Free-standing mesoporous silica films with tunable chiral nematic structures, Nature 468(7322) (2010) 422-5.

[45] J. Xu, T.-D. Nguyen, K. Xie, W.Y. Hamad, M.J. MacLachlan, Chiral nematic porous germania and germanium/carbon films, Nanoscale 7 (31) (2015) 13215-13223.

[46] P.X. Wang, W.Y. Hamad, M.J. MacLachlan, Polymer and Mesoporous Silica Microspheres with Chiral Nematic Order from Cellulose Nanocrystals, Angew. Chem. Int. Ed. Engl. 55(40) (2016) 12460-4.

[47] G.R. Meseck, A.S. Terpstra, A.J. Marenco, S. Trudel, W.Y. Hamad, M.J. MacLachlan, Chiral nematic mesoporous magnetic ferrites, J. Mater. Chem. C 4(48) (2016) 1138211386.

[48] M. Giese, L.K. Blusch, M.K. Khan, M.J. MacLachlan, Functional Materials from Cellulose-Derived Liquid-Crystal Templates, Angew. Chem. Int. Ed. 54(10) (2015) 28882910.

[49] K.E. Shopsowitz, J.A. Kelly, W.Y. Hamad, M.J. MacLachlan, Biopolymer Templated Glass with a Twist: Controlling the Chirality, Porosity, and Photonic Properties of Silica with Cellulose Nanocrystals, Adv. Funct. Mater. 24(3) (2014) 327-338. 
[50] M. Schlesinger, M. Giese, L.K. Blusch, W.Y. Hamad, M.J. MacLachlan, Chiral nematic cellulose-gold nanoparticle composites from mesoporous photonic cellulose, Chem. Commun. 51(3) (2015) 530-3.

[51] A. Espinha, G. Guidetti, M.C. Serrano, B. Frka-Petesic, A.G. Dumanli, W.Y. Hamad, A. Blanco, C. Lopez, S. Vignolini, Shape Memory Cellulose-Based Photonic Reflectors, ACS Appl. Mater. Interfaces 8(46) (2016) 31935-31940.

[52] E. Lizundia, T.-D. Nguyen, Jose L. Vilas, W.Y. Hamad, M.J. MacLachlan, Chiroptical, morphological and conducting properties of chiral nematic mesoporous cellulose/polypyrrole composite films, J. Mater. Chem. A 5(36) (2017) 19184-19194.

[53] G. Guidetti, S. Atifi, S. Vignolini, W.Y. Hamad, Flexible Photonic Cellulose Nanocrystal Films, Adv. Mater. 28(45) (2016) 10042-10047.

[54] T. Hiratani, W.Y. Hamad, M.J. MacLachlan, Transparent Depolarizing Organic and Inorganic Films for Optics and Sensors, Adv. Mater. 29(13) (2017).

[55] Y. Cao, W.Y. Hamad, M.J. MacLachlan, Broadband Circular Polarizing Film Based on Chiral Nematic Liquid Crystals, Adv. Opt. Mater. (2018) 1800412.

[56] A. Matranga, S. Baig, J. Boland, C. Newton, T. Taphouse, G. Wells, S. Kitson, Biomimetic Reflectors Fabricated Using Self-Organising, Self-Aligning Liquid Crystal Polymers, Adv. Mater. 25(4) (2013) 520-523.

[57] M. Mitov, N. Dessaud, Going beyond the reflectance limit of cholesteric liquid crystals, Nat. Mater. 5(5) (2006) 361-364.

[58] A.P. Manning, M. Giese, A.S. Terpstra, M.J. MacLachlan, W.Y. Hamad, R.Y. Dong, C.A. Michal, NMR of guest-host systems: 8CB in chiral nematic porous glasses, Magn. Reson. Chem. 52(10) (2014) 532-539. 
[59] J.A. Kelly, A.M. Shukaliak, C.C.Y. Cheung, K.E. Shopsowitz, W.Y. Hamad, M.J. MacLachlan, Responsive Photonic Hydrogels Based on Nanocrystalline Cellulose, Angew. Chem. Int. Ed. 52(34) (2013) 8912-8916.

[60] M. Giese, L.K. Blusch, M.K. Khan, W.Y. Hamad, M.J. MacLachlan, Responsive mesoporous photonic cellulose films by supramolecular cotemplating, Angew. Chem. Int. Ed. Engl. 53(34) (2014) 8880-4.

[61] M.K. Khan, W.Y. Hamad, M.J. Maclachlan, Tunable mesoporous bilayer photonic resins with chiral nematic structures and actuator properties, Adv. Mater. 26(15) (2014) 2323-8. 